Michel Brion · Roy Joshua

\title{
Intersection cohomology of reductive varieties
}

Received October 8, 2003 and in revised form January 27, 2004

\begin{abstract}
We extend the methods developed in our earlier work to algorithmically compute the intersection cohomology Betti numbers of reductive varieties. These form a class of highly symmetric varieties that includes equivariant compactifications of reductive groups. Thereby, we extend a well-known algorithm for toric varieties.
\end{abstract}

\section{Introduction}

In this paper we extend the methods developed in our previous work [BJ] to algorithmically compute the local and global intersection cohomology Betti numbers of a large class of varieties with group action, that includes toric varieties.

Let $G$ be a complex connected reductive algebraic group and let $B$ be a Borel subgroup. A normal complex algebraic variety $X$, equipped with an action of $G$, is spherical if it contains a dense orbit of $B$. We say that $X$ is scs (simply-connected spherical) if, in addition, the $B$-isotropy group of this dense orbit is connected. Equivalently, any $B$ equivariant finite surjective morphism from a $B$-variety to $X$ is an isomorphism.

For example, the group $G$ is an scs variety with respect to the action of $G \times G$ by left and right multiplication; thus, all normal $G \times G$-equivariant embeddings of $G$ are scs as well. In particular, all toric varieties are scs. Other examples include the space of all skew bilinear forms in $n$ variables for the natural action of $\mathrm{GL}_{n}$ or $\mathrm{SL}_{n}$, and its subvarieties of forms of rank at most $r$. But the space of symmetric bilinear forms in $n$ variables is spherical, not scs.

Spherical $G$-varieties enjoy the following properties:

- they contain only finitely many $B$-orbits, and hence only finitely many $G$-orbits,

The second author is supported by the IHES, MPI (Bonn) and a grant from the National Security Agency.

M. Brion: Institut Fourier, B.P. 74, 38402 Saint Martin d'Hères Cedex, France; e-mail: Michel.Brion@ujf-grenoble.fr

R. Joshua: Department of Mathematics, Ohio State University, Columbus, OH 43210, USA;

e-mail: joshua@math.ohio-state.edu

Current address: School of Mathematics, Institute for Advanced Study, Princeton, NJ 08540, USA; e-mail: joshua@ias.edu

Mathematics Subject Classification (2000): Primary 14F99, 19D99 
- each $G$-orbit admits a slice (see 2.2 below) which is an affine spherical variety under a connected reductive subgroup of $G$, and

- the associated link (see 2.3 below) is a projective spherical variety, of strictly smaller dimension.

Important additional features of scs varieties are

- the connectedness of $B$ - and $G$-isotropy groups of all points, and

- the fact that all slices and links are scs as well.

This makes scs varieties particularly suited for applying the methods from [BJ]. They yield two recursive relations, expressing the global intersection cohomology Betti numbers of projective scs varieties in terms of the corresponding local numbers, and the latter in terms of the global numbers of the links. This is the content of our main result.

Theorem 1.1. Let $X$ be a projective scs $G$-variety; let $I P_{X}(t)$ (resp. I $\left.P_{X, x}(t)\right)$ be the Poincaré polynomial for global intersection cohomology (for the stalks of the intersection cohomology sheaves at $x \in X$, respectively). Then

$$
I P_{X}(t)=\sum_{x}\left(t^{2}-1\right)^{r-r_{x}} \frac{P_{G / T}(t)}{P_{G_{x} / T_{x}}(t)} I P_{X, x}(t)
$$

(sum over representatives of $G$-orbits in $X$ ), and

$$
I P_{X, x}(t)=I P_{\mathcal{S}_{x}, x}(t)=\tau_{\leq d_{x}-1}\left(\left(1-t^{2}\right) I P_{\mathbb{P}\left(S_{x}\right)}(t)\right) .
$$

Here $T$ is a maximal torus of $G$, of dimension $r ; T_{x}$ is a maximal torus of $G_{x}$, of dimension $r_{x} ; \mathcal{S}_{x}$ is a slice at $x$ to $G x$, of dimension $d_{x}, \mathbb{P}\left(\mathcal{S}_{x}\right)=\left(S_{x}-x\right) / \mathbb{G}_{m}$ is the corresponding link for an attractive action of the multiplicative group, and $\tau_{\leq d_{x}-1}$ denotes the truncation to degrees $\leq d_{x}-1$.

(In fact, the Poincaré polynomial $P_{G_{x} / T_{x}}(t)$ divides $P_{G / T}(t)$, and the quotient has nonnegative coefficients. See [BP, p. 321].)

To turn these recursive relations into an algorithm for computing the intersection cohomology Betti numbers, we need a combinatorial description of all isotropy groups, slices, and links. But such a description is unknown in general; in fact, a classification of spherical homogeneous spaces is only known for $G$ of type $A$ (see [Lu]).

Here we obtain such a combinatorial description for the subclass of reductive varieties introduced in [AB1], [AB2]. It contains all normal, $G \times G$-equivariant embeddings of the group $G$, and all their invariant subvarieties. Further, both classes of reductive varieties and of group embeddings are stable under taking slices and links. Our main tool is the classification of reductive varieties in terms of certain toric varieties with additional symmetries, established in [AB1], [AB2]. The resulting algorithm specializes to the one in [St1], [DeLo] and [Fi] for toric varieties.

The latter algorithm defines remarkable numerical invariants of rational polytopes, which in fact make sense for non-rational polytopes as well; see [St1], [St2]. This has been the starting point for several recent investigations, constructing a combinatorial intersection theory for non-rational polytopes (see [BBFK1], [BBFK2], [BBFK3], [Ka] and 
$[\mathrm{BL}])$. It would be interesting to generalize these constructions to the setting of reductive varieties.

The outline of the paper is as follows. Section 2 introduces notation and basic definitions concerning varieties with algebraic group actions, and (equivariant) intersection cohomology. In Section 3, we obtain a variant of a result of [BJ] that applies to all spherical varieties, and we study orbits, slices, and links in scs varieties. Theorem 1.1 is proved in Section 4, and the combinatorics of reductive varieties are developed in Section 5. The final Section 6 is devoted to a few examples, including the case of toric varieties.

\section{Notation and conventions}

2.1. We recall the terminology and conventions from our earlier paper [BJ]. Throughout this paper, we consider complex algebraic varieties, that is, separated reduced schemes of finite type over $\mathbb{C}$. Observe that varieties need not be irreducible; however, we assume them to be equidimensional.

We denote by $G$ a complex linear algebraic group, and by $G^{0}$ its connected component containing the identity. A variety $X$ provided with an algebraic action of $G$ is called a $G$ variety. If, in addition, $X$ admits an equivariant embedding into the projectivization of a $G$-module, we say that $X$ is $G$-quasi-projective. We only consider $G$-varieties where each orbit admits an open $G$-quasi-projective neighborhood. This assumption holds e.g. for normal varieties (see $[\mathrm{Su}]$ for connected $G$, and [Jo] for arbitrary $G$ ).

2.2. Consider a $G$-variety $X$ and a point $x \in X$; let $G x$ be its $G$-orbit and $G_{x}$ its isotropy group. A slice to $G x$ at $x$ is a locally closed subvariety $\mathcal{S}_{x}$ of $X$ containing $x$ and satisfying the following conditions:

(i) $\mathcal{S}_{x}$ is invariant under a maximal torus $T_{x}$ of $G_{x}$.

(ii) The map $G \times \mathcal{S}_{x} \rightarrow X,(g, s) \mapsto g s$, is smooth at the point $(e, x)$, and the dimension of $\mathcal{S}_{x}$ is the codimension of $G x$ in $X$.

Such a slice $\mathcal{S}_{x}$ always exists, and may be chosen invariant under a maximal reductive subgroup of $G_{x}$. Moreover, by shrinking $\mathcal{S}_{x}$ if necessary, we may assume that the map $G \times \mathcal{S}_{x} \rightarrow X$ is smooth everywhere, and that $\mathcal{S}_{x}$ is affine.

2.3. Let $T$ denote a torus acting on a variety $X$ with a fixed point $x$. We say that $x$ is attractive if there exists a one-parameter subgroup $\lambda: \mathbb{G}_{m} \rightarrow T$ such that, for all $y$ in a Zariski neighborhood of $x$, we have $\lim _{t \rightarrow 0} \lambda(t) y=x$. Equivalently, all weights of $T$ acting on the Zariski tangent space at $x$ are contained in an open half-space.

In the situation of 2.2, we say that $\mathcal{S}_{x}$ is an attractive slice if $x$ is an attractive fixed point for the action of $T_{x}$ on $\mathcal{S}_{x}$. (See [BJ, (A.1)] for further details on attractive fixed points.) In this case, the geometric quotient

$$
\mathbb{P}\left(\mathcal{S}_{x}\right)=\left(\mathcal{S}_{x}-x\right) / \mathbb{G}_{m}
$$

exists and we call it the link at $x$. This is a projective variety, since $\mathcal{S}_{x}$ is assumed to be affine. 
2.4. Let $X$ be a variety. We denote by $H^{*}(X)$ the cohomology ring of $X$ with rational coefficients. $I H^{*}(X)$ denotes the corresponding intersection cohomology, for the middle perversity and rational coefficients. $H_{G}^{*}(X)$ (resp. $\left.I H_{G}^{*}(X)\right)$ denotes the corresponding equivariant cohomology ring of $X$ with rational coefficients (the equivariant intersection cohomology of $X$ with rational coefficients, respectively).

For the sake of completeness we briefly recall the definition of the intersection cohomology complex (for the middle perversity). Let $X$ be of dimension $d$ and be provided with a filtration $U_{0} \stackrel{j_{0}}{\rightarrow} U_{1} \stackrel{j_{1}}{\rightarrow} \ldots \stackrel{j_{d-2}}{\rightarrow} U_{d-1} \stackrel{j_{d-1}}{\rightarrow} U_{d}=X$ with each $U_{i}$ open and each $U_{i}-U_{i-1}$ smooth. Then the intersection cohomology complex is the complex of sheaves

$$
I C(X)=\tau_{\leq d-1} R j_{d-1 *} \cdots \tau_{\leq 0} R j_{0 *}(\underline{\mathbb{Q}})
$$

on $X$, where $\mathbb{Q}$ denotes the constant sheaf on $U_{0}$.

Assume, in addition, that $X$ is a provided with a $G$-action and that each $U_{i}$ is $G$ invariant; let $E G \rightarrow B G$ be a universal principal $G$-bundle. Then $E G \underset{G}{\times} U_{i} \stackrel{j_{i}^{G}}{\longrightarrow} E G \times$ $U_{i+1}$ provides a filtration of $E G \underset{G}{\times} X$. Now the equivariant intersection cohomology complex is

$$
I C^{G}(X)=\tau_{\leq d-1} R j_{d-1 *}^{G} \cdots \tau_{\leq 0} R j_{0 *}^{G}(\underline{\mathbb{Q}}),
$$

where $\underline{\mathbb{Q}}$ now denotes the constant sheaf on $E G \underset{G}{\times} U_{0}$. The equivariant intersection cohomology $I H_{G}^{*}(X)$ is defined to be the hypercohomology $\mathbb{H}^{*}\left(E G \underset{G}{\times} X ; C^{G}(X)\right)$. These are discussed in more detail in [B], Section 1]. Both $H_{G}^{*}(X)$ and $I H_{G}^{*}(X)$ are graded modules over $H^{*}(B G)$, the equivariant cohomology ring of the point.

For any integer $n$, we denote by $\mathcal{H}^{n}(I C(X))$ the $n$-th cohomology sheaf of the intersection cohomology complex on $X$. The stalk of the sheaf $\mathcal{H}^{n}(I C(X))$ at a point $x$ is denoted $\mathcal{H}^{n}(I C(X))_{x}$, while the local intersection cohomology with support in $x$ is denoted $I H_{x}^{n}(X)$. They are related as follows: $I H_{x}^{n}(X)$ is the dual space of $\mathcal{H}^{n}(I C(X))_{x}[2 d]$, where $d$ denotes the (complex) dimension of $X$.

\section{The key methods}

3.1. We begin by recalling one of the main results of $[\overline{\mathrm{BJ}}]$ (Theorem 2$)$.

Theorem 3.1. Let $X$ be a $G$-variety containing only finitely many orbits, each of which admits an attractive slice. Then the following hold.

(i) The $H^{*}(B G)$-module $I H_{G}^{*}(X)$ admits a filtration with subquotients $I H_{\mathcal{O}, G}^{*}(X)$, where $\mathcal{O}$ runs through the $G$-orbits in $X$, and $I H_{\mathcal{O}, G}^{*}(X)$ denotes the equivariant intersection cohomology with supports in $\mathcal{O}$.

(ii) For $\mathcal{O}=G x$, the group of components $G_{x} / G_{x}^{0}$ acts on $H^{*}\left(B G_{x}^{0}\right)$ and on $I H_{x}^{*}(X)$, and one obtains the isomorphism

$$
I H_{\mathcal{O}, G}^{*}(X) \cong I H_{x, G_{x}}^{*+2 \operatorname{dim} \mathcal{O}}(X) \cong\left(H^{*+2 \operatorname{dim} \mathcal{O}}\left(B G_{x}^{0}\right) \otimes I H_{x}^{*}(X)\right)^{G_{x} / G_{x}^{0}} .
$$


One may interpret statement (i) as saying that the stratification by orbits is perfect for equivariant intersection cohomology, under the hypotheses of Theorem 3.1 . However, these hypotheses are generally not satisfied by spherical varieties. For example, the linear space $\mathbb{C}^{n}$ is spherical under the natural action of the group $G=\mathrm{SL}_{n}(\mathbb{C})$, and the fixed point 0 admits no attractive neighborhood. Likewise, the space of all $n \times n$ matrices of rank at most 1 is a reductive variety for the same group (acting by left and right multiplication), but again, the fixed point 0 admits no attractive neighborhood.

For this reason, we will obtain a variant of Theorem 3.1 where the existence of attractive slices is replaced by the vanishing of local intersection cohomology groups in all odd degrees. The latter assumption holds for all spherical varieties by [BJ, Theorem 4]; another proof of that theorem will be given in Remark 4.3

Theorem 3.2. Let $X$ denote a $G$-variety containing only finitely many orbits. Assume, in addition, that $H_{x}^{n}(X)=0$ for all $x \in X$ and all odd $n$. Then the conclusions of Theorem 3.1 hold.

Proof. We first prove (ii). Let $i_{\mathcal{O}}: \mathcal{O} \rightarrow X$ denote the inclusion. Then we obtain

$$
I H_{\mathcal{O}, G}^{*}(X) \cong \mathbb{H}^{*}\left(\mathcal{O} ; R i_{\mathcal{O}}^{!} I C^{G}(X)\right) \cong \mathbb{H}^{*}\left(B G_{x} ; R i_{\mathcal{O}}^{!} I C^{G}(X)\right),
$$

where the last isomorphism follows from [BJ, (1.6.1)]. Denoting by $i_{x}: x \rightarrow X$ the inclusion, we also have

$$
R i_{\mathcal{O}}^{!} I C^{G}(X) \cong R i_{x}^{!} I C^{G_{x}}(X)[2 \operatorname{dim}(\mathcal{O})]
$$

by [BJ, (1.6.2)]. This yields an isomorphism

$$
I H_{\mathcal{O}, G}^{*}(X) \cong \mathbb{H}^{*+2 \operatorname{dim} \mathcal{O}}\left(B G_{x} ; R i_{x}^{!} I C^{G_{x}}(X)\right) .
$$

On the other hand, it follows from [BJ] Theorem 1] that the group $G_{x} / G_{x}^{0}$ acts on $\mathbb{H}^{*}\left(B G_{x}^{0}, R i_{x}^{!} I C^{G_{x}^{0}}(X)\right)$, and that

$$
\mathbb{H}^{*}\left(B G_{x} ; R i_{x}^{!} I C^{G_{x}}(X)\right) \cong \mathbb{H}^{*}\left(B G_{x}^{0} ; R i_{x}^{!} I C^{G_{x}^{0}}(X)\right)^{G_{x} / G_{x}^{0}}
$$

Since $B G_{x}^{0}$ is simply connected, the cohomology sheaves of the complex $R i_{x}^{!} I C^{G_{x}^{0}}(X)$ are constant, with stalks $I H_{x}^{n}(X)$. By assumption, these stalks vanish in all odd degrees; it follows that $R i_{x}^{!} I C^{G_{x}^{0}}(X)$ is isomorphic in $D_{b}\left(B G_{x}^{0}\right)$ to its cohomology, $\bigoplus_{n} I H_{x}^{n}(X)[-n]$. This yields an isomorphism

$$
\mathbb{H}^{*}\left(B G_{x}^{0} ; R i_{x}^{!} I C^{G_{x}^{0}}(X)\right) \cong H^{*}\left(B G_{x}^{0}\right) \otimes I H_{x}^{*}(X) .
$$

Thus, $G_{x} / G_{x}^{0}$ acts on the right-hand side. By [BJ] Lemma 3.6], it follows that $G_{x} / G_{x}^{0}$ acts on $I H_{x}^{*}(X)$ so that the isomorphism 3.1 .2 is equivariant. This completes the proof of (ii).

Since $H^{*}\left(B G_{x}^{0}\right)$ vanishes in all odd degrees as well, it also follows that $I H_{G, \mathcal{O}}^{*}(X)$ vanishes in all odd degrees. Now choose $\mathcal{O}$ closed in $X$; then the long exact sequence

$$
\cdots \rightarrow I H_{\mathcal{O}, G}^{n}(X) \rightarrow I H_{G}^{n}(X) \rightarrow I H_{G}^{n}(X-\mathcal{O}) \rightarrow \cdots
$$

breaks up into short exact sequences. This implies (i) by a straightforward induction. 
3.2. Next we review the local structure of spherical varieties. Let $G$ be a connected reductive group and $B$ a Borel subgroup with unipotent radical $U$. We denote by $X$ a spherical $G$-variety, and by rk $X$ its rank, that is, the minimal codimension of a $U$-orbit; then $\mathrm{rk} X$ is the codimension of $U \xi$, for any point $\xi$ of the open $B$-orbit.

Choose a $G$-orbit $\mathcal{O} \subseteq X$; then $\mathcal{O}$ is spherical as well, hence we may choose $x \in \mathcal{O}$ such that $B x$ is open in $G x=\mathcal{O}$. Now let

$$
X_{0}=\{\xi \in X \mid x \in \overline{B \xi}\}=\{\xi \in X \mid \mathcal{O} \subseteq \overline{B \xi}\}
$$

Then $X_{0}$ is an open affine $B$-invariant subset of $X$, intersecting $\mathcal{O}$ along $B x$. Let $P$ be the normalizer of $X_{0}$ in $G$. This is a parabolic subgroup of $G$ containing $B$; let $R_{u}(P)$ be its unipotent radical. Now there exists a Levi subgroup $L$ of $P$ and a closed subvariety $\Sigma$ of $X_{0}$ such that:

- $\Sigma$ is $L$-invariant and contains $x_{0}$, and

- the map $R_{u}(P) \times \Sigma \rightarrow X_{0},(g, \xi) \mapsto g \xi$, is an isomorphism.

Thus, $\Sigma$ is an affine spherical $L$-variety, of rank equal to that of $X$. Moreover, $\Sigma \cap G x$ equals $L x$; this is the unique closed $L$-orbit in $\Sigma$. Finally, the isotropy group $P_{x}$ equals $L_{x}$, and contains the derived subgroup $[L, L]$. (See e.g. $[\overline{\mathrm{Kn}}]$.)

It follows that $P$ is the normalizer of $B x$ in $G$. Further, $L_{x}=[L, L] C_{x}$ where $C$ is the connected center of $L$, and $B_{x}=(B \cap[L, L]) C_{x}$. As a consequence, $L_{x}^{0}$ is a connected reductive group, with Borel subgroup $B \cap L_{x}^{0}=(B \cap[L, L]) C_{x}^{0}=B_{x}^{0}$, of unipotent radical $U \cap L_{x}^{0}=U \cap[L, L]=U_{x}$.

By a corollary of Luna's slice theorem, there exists a closed $L_{x}$-invariant subvariety $\mathcal{S}_{x}$ of $\Sigma$, containing $x$, such that the canonical map

$$
L \times{ }^{L_{x}} \mathcal{S}_{x} \rightarrow \Sigma
$$

is an isomorphism. As a consequence, $\mathcal{S}_{x}$ is a slice to $B x$ at $x$, for the $B$-action on $X$.

Lemma 3.3. $\mathcal{S}_{x}$ is an affine $L_{x}^{0}$-spherical variety, and $\operatorname{rk} \mathcal{S}_{x}=\operatorname{rk} X-\operatorname{rk} G x$.

Proof. Since $L x$ is the unique closed $L$-orbit in $\Sigma$, the point $x$ is the unique closed $L_{x^{-}}$ orbit in $\mathcal{S}_{x}$. In particular, $\mathcal{S}_{x}$ is connected.

We claim that $\mathcal{S}_{x}$ is normal. To see this, consider the normalization $v: \tilde{\mathcal{S}}_{x} \rightarrow \mathcal{S}_{x}$. Then the $L_{x}$-action on $\mathcal{S}_{x}$ lifts to an action on $\tilde{\mathcal{S}}_{x}$ so that $v$ is equivariant. Thus, $v$ extends to a morphism

$$
L \times{ }^{L_{x}} v: L \times^{L_{x}} \tilde{\mathcal{S}}_{x} \rightarrow L \times{ }^{L_{x}} \mathcal{S}_{x}=\Sigma .
$$

Moreover, the morphism $L x^{L_{x}} v$ is finite and birational, since $v$ is. But $\Sigma$ is normal, so that $L \times{ }^{L_{x}} v$ is an isomorphism; thus, the same holds for $v$.

Since $\mathcal{S}_{x}$ is connected and normal, it is irreducible. And since $\Sigma=L \times{ }^{L_{x}} \mathcal{S}_{x}$ contains a dense orbit of $B \cap L$, it follows that $\mathcal{S}_{x}$ contains a dense orbit of $B \cap L_{x}$, and hence of its subgroup of finite index $B \cap L_{x}^{0}$. Thus, $\mathcal{S}_{x}$ is a spherical $L_{x}^{0}$-variety; the assertion on ranks follows from the equalities $\operatorname{rk} X=\operatorname{rk} \Sigma$ and $\operatorname{rk} G x=\operatorname{dim} L x=\operatorname{dim} L / L_{x}$. 
Next we obtain a slight refinement of a result of Knop ([ $\mathrm{Kn}$, Corollary 7.9 and Remark, p. 326]).

Lemma 3.4. There exists an attractive $\mathbb{G}_{m}$-action on $\mathcal{S}_{x}$ that fixes $x$ and commutes with the $L_{x}$-action. As a consequence, $L_{x}$ acts on the link $\mathbb{P}\left(\mathcal{S}_{x}\right)=\left(\mathcal{S}_{x}-x\right) / \mathbb{G}_{m}$, which is a spherical $L_{x}^{0}$-variety of rank equal to $\mathrm{rk} \mathcal{S}_{x}-1$.

Proof. We use the notation of [ $[\mathrm{Kn}$, Section 7]. Notice that the sources of the spherical $L$ variety $\Sigma$ are precisely the closed $L$-invariant subvarieties; in particular, the closed orbit $L x$ is a source. Thus, the closure $\bar{A}_{\Sigma}$ of a generic twisted flat meets $L x$. The normalization of $\bar{A}_{\Sigma}$ is an affine embedding of a finite quotient of the torus $A_{\Sigma}$; let $\mathcal{C}$ be the corresponding cone, then $\mathcal{C}$ is invariant under the little Weyl group $W_{\Sigma}$. By the argument of [ $\mathrm{Kn}, 7.9]$, there exists a $W_{\Sigma}$-invariant one-parameter subgroup $v_{0}$ in the relative interior of $\mathcal{C}$; then both $v_{0}$ and $-v_{0}$ identify to $L$-invariant valuations of the function field $\mathbb{C}(\Sigma)$. Thus, $v_{0}$ yields a $\mathbb{G}_{m}$-action on $\Sigma$ commuting with the $L$-action, that is, an $L$-invariant grading of the algebra of regular functions $\mathbb{C}[\Sigma]$. For this $\mathbb{G}_{m}$-action, $\lim _{t \rightarrow 0} t \xi$ exists and belongs to $L x$ for generic $\xi \in \Sigma$ (since this holds for all $\xi$ in a generic flat, by definition of $v_{0}$ ). It follows that the corresponding grading of $\mathbb{C}[\Sigma]$ is non-negative, and that $\mathbb{C}[\Sigma]_{0}=\mathbb{C}[L x]$. Thus, the grading induces a positive grading of $\mathbb{C}\left[\mathcal{S}_{x}\right]$, the quotient of $\mathbb{C}[\Sigma]$ by the ideal generated by the maximal ideal of $x$ in $\mathbb{C}[L x]$; this positive grading is clearly $L_{x}$-invariant. This proves the first assertion.

From that assertion and Lemma 3.3, it follows that $\mathbb{P}\left(\mathcal{S}_{x}\right)$ is a spherical $L_{x}^{0}$-variety. To determine its rank, choose $\xi \in \mathcal{S}_{x}$ such that $B_{x}^{0} \xi$ is open in $\mathcal{S}_{x}$, and let [ $\xi$ ] be its image in $\mathbb{P}\left(\mathcal{S}_{x}\right)$. Then the isotropy group $B_{[\xi]}$ acts on the orbit $\mathbb{G}_{m} \xi \cong \mathbb{C}^{*}$ via a character with kernel $B_{\xi}$. This implies $U_{[\xi]}=U_{\xi}$, and hence

$$
\operatorname{rk} \mathbb{P}\left(\mathcal{S}_{x}\right)=\operatorname{dim} \mathbb{P}\left(\mathcal{S}_{x}\right)-\operatorname{dim} U_{x}[\xi]=\operatorname{dim} \mathcal{S}_{x}-1-\operatorname{dim} U_{x} \xi=\operatorname{rk} \mathcal{S}_{x}-1
$$

We will also need the following preliminary result.

Lemma 3.5. Let $\xi$ be a point of the open $B \cap L_{x}^{0}$-orbit in $\mathcal{S}_{x}$. Then the orbit $B \xi$ is open in $X$, the isotropy group $B_{\xi}$ is contained in $B_{x}$, and the quotient $B_{x} / B_{\xi}$ is irreducible.

Proof. By the structure of $X_{0}$, we know that $B \xi$ is open in $X$, and $B \xi=B \cap L \xi$. Moreover, since there is an $L$-equivariant map $\Sigma \rightarrow L / L_{x}$, and since $L / L_{x}=(B \cap L) /\left(B \cap L_{x}\right)$, it follows that $B_{\xi} \subseteq B \cap L_{x}=B_{x}$. Note also that the homogeneous space $B_{x} / B_{\xi}$ is the open $B_{x}$-orbit in $\mathcal{S}_{x}$. But $\mathcal{S}_{x}$ is irreducible, so that $B_{x} / B_{\xi}$ is irreducible as well.

3.3. We may now establish the properties of scs varieties presented in the Introduction.

Lemma 3.6. Let $X$ be an scs $G$-variety. Then all $G$-orbits, slices, and links in $X$ are scs, and the $G$-isotropy groups of all points are connected.

Proof. Let $\xi \in X$ be such that $B \xi$ is open in $X$. Then the product $B G_{\xi}$ is open in $G$, so that $G_{\xi} B / B$ is open in $G / B$, and hence is irreducible. But $G_{\xi} B / B \cong G_{\xi} / B_{\xi}$, and $B_{\xi}$ is connected by assumption. Thus, $G_{\xi}$ is connected as well. 
Next consider a $G$-orbit $\mathcal{O}$ in $X$. Choose $x, \xi$ as in Lemma 3.5, the irreducibility of $B_{x} / B_{\xi}$ implies that $B_{x}$ is connected. Hence $L_{x}=[L, L] B_{x}$ is connected as well. Thus, the orbit $G x$ and the slice $\mathcal{S}_{x}$ are scs. It remains to show that the $\operatorname{link} \mathbb{P}\left(\mathcal{S}_{x}\right)$ is scs. Let $\xi$ be as above; then, as noted in the proof of Lemma 3.4 the isotropy group $B_{[\xi]}$ of the corresponding point of $\mathbb{P}\left(\mathcal{S}_{x}\right)$ is the kernel of a character of $B \xi$. This character is surjective, since $\operatorname{dim} B_{[\xi]}=\operatorname{dim} B-\operatorname{dim} B[\xi]=\operatorname{dim} B-\operatorname{dim} B \xi+1=\operatorname{dim} B_{\xi}+1$. But $B_{\xi}$ is connected, so that $B_{[\xi]}$ is connected.

Lemma 3.7. Let $X$ be an scs $G$-variety. Then the B-isotropy groups of all points are connected.

Proof. Let $\xi \in X$. By Lemma 3.6, we may assume that $X=G \xi$. We argue by induction on the codimension $c$ of $B \xi$ in $X$.

If $c=0$, then $B \xi$ is open in $X$, so that $B_{\xi}$ is connected by assumption. If $c \geq 1$, then we may find a minimal parabolic subgroup $P \supset B$ such that $\overline{B \xi}$ is not $P$-invariant. Then $B \xi$ is contained in $P \xi$ as a closed $B$-orbit of codimension 1 , and $P \xi$ contains an open $B$-orbit, say $B \eta$, of dimension $\operatorname{dim} B \xi+1$. Thus, $B_{\eta}$ is connected by the induction assumption. Further, $P_{\eta} / B_{\eta} \cong P_{\eta} B / B$, and $P_{\eta} B$ is open in $P$ (since $B \eta$ is open in $P \eta=P \xi)$, so that $P_{\eta} / B_{\eta}$ is irreducible. Thus, $P_{\eta}$ is connected as well.

On the other hand, the natural map $P \times{ }^{B} B \xi \rightarrow P \xi$ is finite, since $P$ moves $\overline{B \xi}$. In other words, $B_{\xi}$ has finite index in $P_{\xi}$. But $P_{\xi}$ is conjugate to $P_{\eta}$, so $B_{\xi}$ is connected.

\section{Proof of the main theorem}

4.1. We begin by introducing various Poincaré series. These are formal power series in a variable $t$, with integer coefficients.

If $G$ is a linear algebraic group, we put

$$
P^{G}(t)=\sum_{n} \operatorname{dim} H^{n}(B G) t^{n},
$$

the Poincaré series of $B G$. For example, if $G$ is a torus of dimension $r$, then

$$
P^{G}(t)=\frac{1}{\left(1-t^{2}\right)^{r}} .
$$

More generally, if $G$ is connected with maximal torus $T$ of dimension $r$, then we have a fibration $B T \rightarrow B G$ with fiber $G / T$ homotopic to the flag manifold of $G$. Hence the cohomology of $G / T$ vanishes in all odd degrees; this implies

$$
P^{G}(t)=\frac{1}{\left(1-t^{2}\right)^{r} P_{G / T}(t)},
$$

where $P_{G / T}(t)$ is the Poincaré polynomial of $G / T$.

If $X$ is a $G$-variety, we put

$$
I P_{X}^{G}(t)=\sum_{n} \operatorname{dim} I H_{G}^{n}(X) t^{n} .
$$


In particular, if $G$ is the trivial group, then $I P_{X}(t)$ is the intersection cohomology Poincaré polynomial of $X$. If $X$ is projective of dimension $d$, then we have $I P_{X}(t)=t^{2 d} I P_{X}(1 / t)$, by Poincaré duality.

If $G$ is connected and $X$ is a projective $G$-variety, then we have

$$
I P_{X}^{G}(t)=P^{G}(t) I P_{X}(t),
$$

as follows from the degeneration of the spectral sequence in equivariant intersection cohomology (see e.g. [BJ], (1.5.2)]).

Returning to an arbitrary $G$-variety $X$, we put, for any $x \in X$,

$$
I P_{x, X}^{G_{x}}(t)=\sum_{n} \operatorname{dim} I H_{x, G_{x}}^{n}(X) t^{n} .
$$

In particular, $I P_{x, X}(t)=\sum_{n} \operatorname{dim} I H_{x}^{n}(X) t^{n}$ is the Poincaré polynomial for local intersection cohomology with support in $x$.

On the other hand, one also has the Poincaré series for the stalks of the equivariant intersection cohomology sheaves:

$$
I P_{X, x}^{G_{x}}(t)=\sum_{n} \operatorname{dim} \mathcal{H}^{n}\left(i_{x}^{*} I C^{G_{x}}(X)\right) t^{n},
$$

where $i_{x}: x \rightarrow X$ denotes the inclusion.

Note that the Poincaré polynomial $I P_{X, x}(t)=\sum_{n} \operatorname{dim} \mathcal{H}^{n}\left(i_{x}^{*} I C(X)\right) t^{n}$ satisfies the Verdier duality

$$
I P_{X, x}(t)=t^{2 d} I P_{x, X}\left(\frac{1}{t}\right) .
$$

4.2. We may now formulate a direct consequence of Theorems 3.1 and 3.2 for these Poincaré series.

Proposition 4.1. (i) Let $X$ be a $G$-variety satisfying the assumptions of Theorem 3.1 or 3.2. Then

$$
I P_{X}^{G}(t)=\sum_{x} t^{-2 \operatorname{dim} G x} I P_{x, X}^{G_{x}}(t)
$$

(sum over representatives of $G$-orbits in $X$ ).

(ii) If, in addition, $G_{x}$ is connected, then

$$
I P_{x, X}^{G_{x}}(t)=P^{G_{x}}(t) I P_{x, X}(t) .
$$

Next let $X$ be a projective scs variety. Then Proposition 4.1 and 4.1 .3 , 4.1.5, 4.1 .8 imply

$$
I P_{X}(t)=\sum_{x}\left(1-t^{2}\right)^{r-r_{x}} \frac{P_{G / T}(t)}{P_{G_{x} / T_{x}}(t)} t^{2 d_{x}} I P_{X, x}\left(\frac{1}{t}\right) .
$$

Replacing $t$ with $1 / t$ and using Poincaré duality for $X, G / T$ and $G_{x} / T_{x}$ yields the equality (1.0.1) in Theorem 1.1 Now the equality 1.0.2 is a consequence of the following statement. 
Proposition 4.2. Consider a $G$-variety $X$, a point $x \in X$, and a slice $\mathcal{S}_{x}$ to $G x$ at $x$. Then

$$
I P_{x, X}(t)=t^{2\left(d-d_{x}\right)} I P_{x, \mathcal{S}_{x}}(t)=t^{2 d} I P_{\mathcal{S}_{x}, x}\left(\frac{1}{t}\right),
$$

where $d=\operatorname{dim} X$ and $d_{x}=\operatorname{codim} G x=\operatorname{dim} \mathcal{S}_{x}$.

If, in addition, $\mathcal{S}_{x}$ is attractive with link $\mathbb{P}\left(\mathcal{S}_{x}\right)$, then

$$
I P_{\mathcal{S}_{x}, x}(t)=\tau_{\leq d_{x}-1}\left(\left(1-t^{2}\right) I P_{\mathbb{P}\left(\mathcal{S}_{x}\right)}(t)\right)
$$

if $d_{x} \geq 2$, and $I P_{\mathcal{S}_{x}, x}(t)=1$ otherwise.

Proof. One obtains a quasi-isomorphism

$$
R i_{x, X}^{!} I C(X) \cong R i_{x, \mathcal{S}_{x}}^{!} I C\left(\mathcal{S}_{x}\right)\left[-2\left(d-d_{x}\right)\right],
$$

where $i_{x, X}: x \rightarrow X$ and $i_{x, \mathcal{S}_{x}}: x \rightarrow \mathcal{S}_{x}$ denote the inclusions. This yields the first equality in 4.2.1; the second one follows from 4.1.8.

For 4.2.2), observe that $\mathcal{H}^{n}\left(I C\left(\mathcal{S}_{x}\right)\right)_{x} \cong I H^{n}\left(\mathcal{S}_{x}\right) \cong I H^{n}\left(\mathcal{S}_{x}-x\right)$ for all $n \leq d_{x}-1$, while $\mathcal{H}^{n}\left(I C\left(\mathcal{S}_{x}\right)\right)_{x}=0$ for all $n>d_{x}-1$ (see, for example, the definition of the intersection cohomology complex in 2.4). Thus, $I P_{\mathcal{S}_{x}, x}(t)=\tau_{\leq d_{x}-1}\left(I P_{\mathcal{S}_{x}-x}(t)\right)$. Now consider the Wang exact sequence

$\cdots \rightarrow I H^{n-2}\left(\mathbb{P}\left(\mathcal{S}_{x}\right)\right) \rightarrow I H^{n}\left(\mathbb{P}\left(\mathcal{S}_{x}\right)\right) \rightarrow I H^{n}\left(\mathcal{S}_{x}-x\right)$

$$
\rightarrow I H^{n-1}\left(\mathbb{P}\left(\mathcal{S}_{x}\right)\right) \rightarrow I H^{n+1}\left(\mathbb{P}\left(\mathcal{S}_{x}\right)\right) \rightarrow \cdots,
$$

where the first and last maps are multiplication by the class of a hyperplane in $\mathbb{P}\left(\mathcal{S}_{x}\right)$ (see e.g. [BJ 3.5]). By the hard Lefschetz theorem in intersection cohomology, these maps are injective for all $n \leq \operatorname{dim} \mathbb{P}\left(S_{x}\right)=\operatorname{dim} \mathcal{S}_{x}-1=d_{x}-1$. Therefore, the Wang exact sequence breaks up into short exact sequences for all $n \leq d_{x}-1$. This yields $\tau_{\leq d_{x}-1}\left(I P\left(\mathcal{S}_{x}-x\right)\right)=\tau_{\leq d_{x}-1}\left(\left(1-t^{2}\right) I P_{\mathbb{P}\left(\mathcal{S}_{x}\right)}(t)\right)$.

Remark 4.3. The results of Sections 3 and 4 yield another proof of the vanishing of $I H^{n}(X)$ and $I H_{x}^{n}(X)$ for all odd $n$ and spherical $X$ ([B], Theorem 4]). Indeed, let us argue by induction on $\operatorname{dim} X=d$. By Lemma 3.4, all links of $X$ are spherical, of dimension $<d$. Thus, by Proposition 4.2 and the induction assumption, $I H_{x}^{n}(X)$ for all odd $n$ and $x \in X$. Now Theorem 3.2 implies the vanishing of $I H^{n}(X)$ for all odd $n$.

Remark 4.4. For any closed connected subgroup $H \subseteq G$, put

$$
P_{G / H}(t)=(-1)^{r-s} \frac{P^{H}(t)}{P^{G}(t)},
$$

where $s$ is the rank of $H$ (and $r$ is the rank of $G$ ). Then $P_{G / H}(t)$ is the virtual Poincaré polynomial of $G / H$, as follows from [DiLe, Theorem 6.1(ii)] applied to the fibration $B H \rightarrow B G$ with fiber $G / H$. In particular, $P_{G / H}(t)$ is a polynomial with rational coefficients, of degree $\operatorname{dim} G / H$, that only depends on the structure of complex algebraic 
variety on $G / H$. (See $[\overline{\mathrm{BP}}]$ for more on virtual Poincaré polynomials of homogeneous spaces.)

With this notation, 4.1 .3 yields

$$
P_{G x}(t)=P_{G / G_{x}}(t)=\left(t^{2}-1\right)^{r-r_{x}} \frac{P_{G / T}(t)}{P_{G_{x} / T_{x}}(t)},
$$

so that $(1.0 .1)$ translates into

$$
I P_{X}(t)=\sum_{x} P_{G x}(t) I P_{X, x}(t) .
$$

We may regard the terms on the right-hand side as virtual Poincare polynomials for intersection cohomology with supports in orbits.

\section{Reductive varieties}

5.1. We first give an overview of affine reductive varieties, after [AB1]. For this, we need to introduce notation concerning reductive groups.

Let $G$ be a connected reductive group and let $B, B^{-}$be opposite Borel subgroups, i.e., $T=B \cap B^{-}$is a maximal torus of $G$; let $U=R_{u}(B), U^{-}=R_{u}\left(B^{-}\right)$be the unipotent radicals of $B, B^{-}$. The character group of $T$ is denoted by $\Lambda$, and called the weight lattice; we put $\Lambda_{\mathbb{R}}=\Lambda \otimes_{\mathbb{Z}} \mathbb{R}$. Let $W$ be the Weyl group of $(G, T)$; it acts on $\Lambda$ and on $\Lambda_{\mathbb{R}}$. The root system of $(G, T)$ is denoted by $\Phi$, with the subsets $\Phi^{+}$of positive roots (the roots of $(B, T)$ ), and $\Pi$ of simple roots.

For a subset $I \subseteq \Pi$, we denote by $\Phi_{I}$ the corresponding subsystem of $\Phi$, and by $W_{I}$ (resp. $P_{I} \supseteq B$ and $P_{I}^{-} \supseteq B^{-}$) the corresponding parabolic subgroup of $W$ (resp. opposite parabolic subgroups of $G$ ). We put $L_{I}=P_{I} \cap P_{I}^{-}$; this is a Levi subgroup of $P_{I}$ and $P_{I}^{-}$, with root system $\Phi_{I}$ and Weyl group $W_{I}$. Let $\ell$ be the length function of $W$, and let $W^{I}$ be the subset of representatives of minimal length of $W / W_{I}$. Then the Poincaré polynomial of $G / P_{I}$ equals $\sum_{w \in W^{I}} t^{2 \ell(w)}$.

Now consider the connected reductive group $G \times G$, with Borel subgroup $B^{-} \times B$ and maximal torus $T \times T$. Let $\operatorname{diag} T$ be the diagonal in $T \times T$. We say that an affine $G \times G$-variety $X$ is reductive (for $G$ ) if it satisfies the following conditions.

(i) $X$ is normal.

(ii) There exists $x \in X$, fixed by $\operatorname{diag} T$, such that the orbit $\left(B^{-} \times B\right) x$ is dense in $X$.

(iii) The isotropy group $(G \times G)_{x}$ is connected.

Further, we may replace (iii) with the assumption of connectedness of $\left(B^{-} \times B\right)_{x}$, or of $(T \times T)_{x}$. Thus, affine reductive varieties are scs $G \times G$-varieties. Note also that the set of all $x \in X$ satisfying (ii) is a unique $T \times T$-orbit. Any such point is called a base point. Also note that $\operatorname{dim}(T \times T) x=\operatorname{rk} X$.

By the Bruhat decomposition, the multiplication of $G$ yields an open immersion $U^{-} \times T \times U \rightarrow G$. Thus, the group $G$, regarded as a $G \times G$-variety via left and right multiplication, is an affine reductive variety. More generally, all affine $G \times G$-equivariant 
embeddings of $G$, or of quotients of $G$ by connected normal subgroups, are reductive varieties for $G$.

Next we summarize results of [AB1] about the classification and orbit structure of affine reductive varieties. Let $X^{\prime}$ be the closure in $X$ of the orbit of base points; this is an affine toric variety for $T$ (identified with $T \times\{1\} \subset G \times G)$. Thus, $X^{\prime}$ is uniquely determined by the set of weights of $T$ in its coordinate ring. Further, this set is the intersection of $\Lambda$ with a uniquely determined rational convex polyhedral cone $\sigma$ in $\Lambda_{\mathbb{R}}$.

The assignment $X \mapsto \sigma$ yields a bijective correspondence from affine reductive varieties to rational convex polyhedral cones $\sigma \subseteq \Lambda_{\mathbb{R}}$ satisfying the following conditions:

(i) The relative interior $\sigma^{0}$ meets $\Lambda_{\mathbb{R}}^{+}$.

(ii) The distinct $w \sigma^{0}(w \in W)$ are disjoint.

We then say that $\sigma$ is a $W$-admissible cone, and put $X=X_{\sigma}$; then $\operatorname{rk} X=\operatorname{dim} \sigma$. The $G \times G$-orbit closures in $X_{\sigma}$ are the affine reductive varieties associated to $W$-admissible faces of $\sigma$.

For any $W$-admissible cone $\sigma$, let $N_{W}(\sigma)$ (resp. $C_{W}(\sigma)$ ) be its normalizer (resp. centralizer) in $W$. Then we have $N_{W}(\sigma)=W_{I}$ and $C_{W}(\sigma)=W_{J}$ for subsets $J=J(\sigma) \subseteq$ $I=I(\sigma) \subseteq \Pi$. Further, all roots in the complement $K=K(\sigma)=I-J$ are orthogonal to $J$, so that $W_{I}=W_{J} \times W_{K}$ and $L_{I}=L_{J} L_{K}$; and $J$ (resp. $K$ ) consists of those simple roots such that the corresponding wall of $\Lambda_{\mathbb{R}}^{+}$contains $\sigma$ (resp. meets $\sigma^{0}$ ).

Let $\Lambda_{\sigma}$ be the subgroup of $\Lambda$ spanned by all elements of $\Lambda \cap \sigma$; this is a saturated sublattice of $\Lambda$, that is, the quotient $\Lambda / \Lambda_{\sigma}$ is torsion-free. Let $T_{\sigma} \subseteq T$ be the intersection of the kernels of all characters in $\Lambda_{\sigma}$ (or, equivalently, in $\Lambda \cap \sigma$ ); this is a central subtorus of $L_{K}$, with character group $\Lambda / \Lambda_{\sigma}$. Put $G_{\sigma}=\left[L_{J}, L_{J}\right] T_{\sigma}$; this is a connected reductive subgroup of $L_{J}$ (denoted by $H_{J}$ in [AB1] ), with maximal torus $T_{\sigma}$. Thus, $G_{\sigma}$ is a normal subgroup of $L_{I}$, and the quotient $L_{I} / G_{\sigma}$ is isomorphic to $L_{K} / T_{\sigma}$. Now

$$
(G \times G)_{x}=\left(R_{u}\left(P_{I}\right) \times R_{u}\left(P_{I}^{-}\right)\right)\left(G_{\sigma} \times G_{\sigma}\right) \operatorname{diag}\left(L_{K}\right),
$$

up to conjugacy by an element of $T \times T$.

As a consequence, any $G \times G$-orbit in an affine reductive variety admits a homogeneous fibration over a product of two opposite flag manifolds, with fiber a connected reductive group. Specifically, we have a $G \times G$-equivariant morphism

$$
(G \times G) x \rightarrow G / P_{I} \times G / P_{I}^{-}
$$

with fiber

$$
\left(L_{I} \times L_{I}\right) /\left(G_{\sigma} \times G_{\sigma}\right) \operatorname{diag} L_{K}=L_{I} / G_{\sigma}=L_{K} / T_{\sigma} .
$$

Here $P_{I} \times P_{I}^{-}$acts on this fiber via its quotient $L_{I} \times L_{I}$ acting on $L_{I} / G_{\sigma}$ by left and right multiplication. Note that $L_{K} / T_{\sigma}$ is a connected reductive group with weight lattice $\Lambda_{\sigma}$ and root system $\Phi_{K}$.

It follows readily that

$$
\operatorname{dim} X_{\sigma}=\operatorname{dim}(G \times G) x=\left|\Phi-\Phi_{J}\right|+\operatorname{dim} \sigma,
$$


and that the virtual Poincaré polynomial of $(G \times G) x$ (see Remark 4.4 ) is given by

$$
P_{(G \times G) x}(t)=\left(t^{2}-1\right)^{\operatorname{dim} \sigma}\left(\sum_{w \in W^{I}} t^{2 \ell(w)}\right)^{2}\left(\sum_{w \in W_{K}} t^{2 \ell(w)}\right) .
$$

As another consequence of (5.1.1), $X$ is an affine embedding of the quotient of $G$ by a connected normal subgroup if and only if $I=\Pi$, that is, $\sigma$ is $W$-invariant. In particular, affine embeddings of $G$ correspond to $W$-invariant cones with non-empty interior.

5.2. Following [AB2, Section 2], we define projective reductive varieties and we sketch how to deduce their main properties from the affine case.

Consider a projective irreducible $G \times G$-variety $X$ equipped with an ample $G \times G$ linearized line bundle $L$. Let $R=\bigoplus_{n=0}^{\infty} \Gamma\left(X, L^{\otimes n}\right)$; this is a graded, finitely generated reduced algebra where $G \times G$ acts. This defines an affine variety $\tilde{X}$ where $\mathbb{G}_{m} \times G \times G$ acts. Further, the action of $\mathbb{G}_{m}$ is attractive, and the corresponding link is nothing but $X$. We say that the pair $(X, L)$ is a linearized projective $G \times G$-variety.

Put $\tilde{G}=\mathbb{G}_{m} \times G$; this is a connected reductive group with weight lattice $\tilde{\Lambda}=\mathbb{Z} \times \Lambda$. We may regard $\tilde{X}$ as a $\tilde{G} \times \tilde{G}$-variety, where $\mathbb{G}_{m} \times \mathbb{G}_{m}$ acts via its morphism $\left(t_{1}, t_{2}\right) \mapsto$ $t_{1} t_{2}^{-1}$ to $\mathbb{G}_{m}$. For any $x \in X$ with representative $\tilde{x} \in \tilde{X}$, we obtain readily an exact sequence of isotropy groups:

$$
1 \rightarrow \mathbb{G}_{m} \rightarrow(\tilde{G} \times \tilde{G})_{\tilde{x}} \rightarrow(G \times G)_{x} \rightarrow 1 .
$$

We say that $X$ is reductive for $G$ if $\tilde{X}$ is reductive for $\tilde{G}$; then $(X, L)$ is called a linearized reductive variety. These may be characterized as those linearized projective $G \times G$-varieties $(X, L)$ that satisfy the following conditions:

(i) $X$ is normal.

(ii) There exists $x \in X$, fixed by $\operatorname{diag} T$, such that the orbit $\left(B^{-} \times B\right) x$ is dense in $X$, and that $\operatorname{diag} T$ fixes the fiber of $L$ at $x$.

(iii) The isotropy group $(G \times G)_{x}$ is connected.

Again, (iii) is equivalent to the assumption of connectivity of $\left(B^{-} \times B\right)_{x}$, or of $(T \times T)_{x}$; and the set of all $x \in X$ satisfying (ii) is a unique $T \times T$-orbit: the orbit of base points, of dimension equal to $\mathrm{rk} X$.

Thus, projective reductive varieties are scs. Again, examples include $G \times G$-equivariant embeddings of the quotient of $G$ by a connected normal subgroup.

Let $\sigma \subseteq \tilde{\Lambda}_{\mathbb{R}}=\mathbb{R} \times \Lambda_{\mathbb{R}}$ be the cone associated to $\tilde{X}$, and put $\delta=\sigma \cap\left(1 \times \Lambda_{\mathbb{R}}\right)$. Then $\delta$ is a lattice polytope in $\Lambda_{\mathbb{R}}$, and $\sigma$ is the cone over $\delta$. Since $\sigma$ is $W$-admissible, $\delta$ satisfies the following conditions:

(i) The relative interior $\delta^{0}$ meets $\Lambda_{\mathbb{R}}^{+}$.

(ii) The distinct translates $w \delta^{0}(w \in W)$ are disjoint.

A lattice polytope $\delta \subset \Lambda_{\mathbb{R}}$ satisfying (i) and (ii) is called a $W$-admissible polytope. These classify polarized reductive varieties; we denote by $\left(X_{\delta}, L_{\delta}\right)$ the linearized reductive variety with polytope $\delta$; then $\operatorname{dim} X_{\delta}=\operatorname{rk} \delta$. The closure in $X$ of the orbit of base points, 
equipped with the restriction of $L$, is the linearized toric variety with polytope $\delta$. The $G \times G$-orbit closures in $X_{\delta}$ are the $X_{\phi}$, where $\phi \subseteq \delta$ is a $W$-admissible face.

Since $N_{W}(\sigma)=N_{W}(\delta)$ and $C_{W}(\sigma)=C_{W}(\delta)$, we obtain two subsets $J=J(\delta) \subseteq$ $I=I(\delta) \subseteq \Pi$ satisfying the properties of the previous subsection. Now the description 5.1.1) of the isotropy group $(G \times G)_{x}$ carries over to this projective setting, with $\Lambda_{\sigma}$ being replaced by the lattice $\Lambda_{\delta}$ spanned by the differences of any two elements of $\Lambda \cap \delta$.

As a consequence, the description of orbits as fibered spaces carries over as well; specifically, the analogues of (5.1.2), (5.1.3, (5.1.4) and 5.1.5) hold with $\sigma$ being replaced by $\delta$. Further, projective embeddings of a quotient of $G$ by a connected normal subgroup (resp. of $G$ ) correspond to $W$-invariant lattice polytopes (resp. with non-empty interior).

5.3. We obtain a combinatorial description of slices and links in reductive varieties. Consider a $W$-admissible polytope $\delta \subset \Lambda_{\mathbb{R}}$, and a $W$-admissible face $\varphi \subseteq \delta$. These correspond to a linearized reductive variety $\left(X_{\delta}, L_{\delta}\right)$ together with a $G \times G$-orbit $\mathcal{O}=$ $\mathcal{O}_{\varphi}$ : the open orbit in $X_{\varphi} \subseteq X_{\delta}$. We describe the local structure of $X$ along $\mathcal{O}$, by making explicit the objects introduced in 3.2 .

Let $x$ be a base point of $\mathcal{O}$; then $\left(B^{-} \times B\right) x$ is open in $(G \times G) x=\mathcal{O}$. Further, it follows from 5.1.1 that the normalizer $P$ of $\left(B^{-} \times B\right) x$ in $G \times G$ equals $P_{J}^{-} \times P_{J}$, where $J=J(\varphi)$. Since $x$ is fixed by $\operatorname{diag} T$, the Levi subgroup $L$ of $P$ equals $L_{J} \times L_{J}$. Further, by [AB1, Lemma 2.8], the variety $\Sigma$ is an affine reductive variety for $L_{J}$; one readily checks that the corresponding $W_{J}$-admissible cone is generated by the differences $\lambda-\mu$, where $\lambda \in \delta$ and $\mu \in \varphi$.

Now by (5.1.1) again, we have $L_{x}=G_{\varphi} \times G_{\varphi}$. Note that $G_{\varphi}$ is a connected reductive subgroup of $G$, normalized by $T$; further, $T_{\varphi}$ is a maximal torus of $G_{\varphi}$, so that the weight lattice of $G_{\varphi}$ equals $\Lambda_{\varphi}=\Lambda / \Lambda_{\varphi}$. The set of simple roots of $G_{\varphi}$ is $J=J(\varphi)$, with Weyl group $W_{J}=C_{W}(\varphi)$; we denote the latter by $W_{\varphi}$.

By [AB1, Lemma 4.1], the slice $\mathcal{S}_{x}$ is an affine reductive variety for $G_{\varphi}$. Denote its $W_{\varphi}$-admissible cone by $\sigma=\sigma_{\varphi}$; this cone is the image in $\Lambda_{\varphi}$ of the cone of $\Sigma$. So we may regard $\sigma$ as the normal cone to $\delta$ along its face $\varphi$. Note the equality $\operatorname{rk} \mathcal{S}_{x}=\operatorname{dim} \delta-\operatorname{dim} \varphi$.

To describe the link $\mathbb{P}\left(\mathcal{S}_{x}\right)$, note first that the closed convex cone $\sigma$ contains no line. Thus, we may find a linear form $f$ on $\Lambda_{\mathbb{R}} / \Lambda_{\varphi, \mathbb{R}}$ that takes positive values at all nonzero points of $\sigma$. We may assume, in addition, that $f$ takes integer values at all points of $\Lambda / \Lambda_{\varphi}$, and is invariant under the normalizer of $\sigma$ in $W_{\varphi}$. Then by [AB1, 3.2, 4.1], $f$ yields a positive $G_{\varphi} \times G_{\varphi}$-invariant grading of the algebra of regular functions on $\mathcal{S}_{x}$. In other words, $f$ defines an attractive $\mathbb{G}_{m}$-action on $\mathcal{S}_{x}$ that commutes with the action of $G_{\varphi} \times G_{\varphi}$. Now $\mathbb{P}\left(\mathcal{S}_{x}\right)$ is the reductive variety for $G_{\varphi}$ associated with the polytope $\sigma \cap(f=n)$, where $n$ is a suitable positive integer. We may regard this polytope as the link of $\delta$ along its face $\varphi$; we have $\operatorname{rk} \mathbb{P}\left(\mathcal{S}_{x}\right)=\operatorname{dim} \delta-\operatorname{dim} \varphi-1$.

If $X_{\delta}$ is an embedding of a quotient of $G$ by a connected normal subgroup, then $\delta$ is $W$-invariant, so that $\sigma_{\varphi}$ is invariant under $W_{\varphi}$. Thus, $\mathcal{S}_{x}$ is an embedding of a quotient of $G_{\varphi}$ by a connected normal subgroup. So the class of embeddings of connected reductive groups is stable under taking slices and, likewise, links. 


\section{Examples}

6.1. We begin with the case of toric varieties, where the objects introduced in Section 5 take a very simple form.

Let $X$ be a projective toric variety; let $T$ be the corresponding torus, with character group $\Lambda$. Then $X$ corresponds to a fan $\Sigma$, consisting of the normal cones to all faces of a lattice polytope $\delta \in \Lambda_{\mathbb{R}}$. (This polytope is not uniquely determined by $X$, but so are the partially ordered set of its faces, and their directions.)

The $T$-orbits in $X$ correspond to cones of $\Sigma$, that is, to faces of $\delta$. For any such face $\varphi$, the isotropy group of the corresponding orbit $\mathcal{O}=\mathcal{O}_{\varphi}$ is a subtorus $T_{\varphi}$ of $T$, the intersection of all characters in the space $\Lambda_{\varphi, \mathbb{R}}$ spanned by the differences $\lambda-\mu$, where $\lambda, \mu \in \varphi$.

Moreover, $\mathcal{O}$ admits an open $T$-invariant neighborhood in $X$, isomorphic to the product $\mathcal{O} \times \mathcal{S}_{\varphi}$, where $\mathcal{S}_{\varphi}$ is an affine toric variety for $T_{\varphi}$ with a fixed point $x_{\varphi}$. The cone associated with $\mathcal{S}_{\varphi}$ is dual to the cone $\sigma_{\varphi}$, image in $\Lambda_{\mathbb{R}} / \Lambda_{\varphi, \mathbb{R}}$ of the cone generated by the differences $\lambda-\mu$, where $\lambda \in \delta, \mu \in \varphi$.

It follows that $\mathcal{S}_{\varphi}$ is an attractive slice at any point of $\mathcal{O}$, and that the associated link $\mathbb{P}\left(\mathcal{S}_{\varphi}\right)$ is a projective toric variety with polytope the link of $\varphi$ in $\delta$. The $x_{\varphi}, \varphi$ a face of $\delta$, form a system of representatives of the $T$-orbits in $X$. (See e.g. [ [Fu, 1.4, 2.1].)

Now Theorem 1.1 yields the equalities

$$
I P_{X}(t)=\sum_{\varphi}\left(t^{2}-1\right)^{\operatorname{dim} \varphi} I P_{X, x_{\varphi}}(t)
$$

(sum over all faces of $\delta$ ), and

$$
I P_{X, x_{\varphi}}(t)=I P_{\mathcal{S}_{\varphi}, x_{\varphi}}(t)=\tau_{\leq \operatorname{codim} \varphi-1}\left(\left(1-t^{2}\right) I P_{\mathbb{P}\left(\mathcal{S}_{\varphi}\right)}(t)\right) .
$$

When expressed in terms of cones of $\Sigma$, 6.1.2 and 6.1.1 give back the main result of [Fi]; see also [DeLo], [St1], [St2].

6.2. Next we describe orbits, slices and links in reductive varieties of rank 1. Indeed, the rank, rather than the dimension, measures how complicated a spherical variety is; and spherical (resp. reductive) varieties of rank 0 are just flag manifolds $G / P$ (resp. products of two opposite flag manifolds $\left.G / P_{I} \times G / P_{I}^{-}\right)$.

By 5.2, a linearized reductive variety $(X, L)$ of rank 1 corresponds to a $W$-admissible line segment $\delta=[\lambda, \mu]$, where $\lambda, \mu \in \Lambda$. We may assume that $\lambda$ is dominant; then it is a $W$-admissible face, corresponding to a closed $G \times G$-orbit $\mathcal{O}=\mathcal{O}_{\lambda}$. With the notation of 5.2, we have $\Lambda_{\lambda}=0$, so that $T_{\lambda}=T$. Further, $I(\lambda)=J(\lambda)$ is the set of simple roots orthogonal to $\lambda$, so that $G_{\lambda}=L_{I(\lambda)}$ is the common Levi subgroup to $P_{I(\lambda)}$ and $P_{I(\lambda)}^{-}$. Moreover, $\mathcal{O}_{\lambda}=G / P_{I(\lambda)} \times G / P_{I(\lambda)}^{-}$. The corresponding slice is the affine reductive variety for $L_{I(\lambda)}$ with cone the ray spanned by $\mu-\lambda$. Set $I(\delta)=I(\lambda) \cap I(\mu)$; then the corresponding link is the product

$$
L_{I(\lambda)} / P_{I(\delta)} \cap L_{I(\lambda)} \times L_{I(\lambda)} / P_{I(\delta)}^{-} \cap L_{I(\lambda)} \cong P_{I(\lambda)} / P_{I(\delta)} \times P_{I(\lambda)}^{-} / P_{I(\delta)}^{-} .
$$


If $\mu$ is also dominant, then $X$ contains three $G \times G$-orbits: the open orbit, and the closed orbits corresponding to $\lambda$ and $\mu$. The isotropy group of a base point of the open orbit $\mathcal{O}_{\delta}$ equals $\left(R_{u}\left(P_{I(\delta)}\right) \times R_{u}\left(P_{I(\delta)}^{-}\right)\right)\left(G_{\delta} \times G_{\delta}\right)$, where $G_{\delta} \subset L_{I(\delta)}$ is the connected kernel of the character $\mu-\lambda$. Thus, $\mathcal{O}_{\delta}$ fibers over $G / P_{I(\delta)} \times G / P_{I(\delta)}^{-}$with fiber $\mathbb{G}_{m}$.

But if $\mu$ is not dominant, then $\lambda$ is the unique $W$-admissible proper face, so that $X$ contains only two orbits. In that case, $\lambda$ and $\mu$ are exchanged by a unique simple reflection $s_{\alpha}$, where $\alpha \in \Pi$, so that $\mu-\lambda$ is a non-zero multiple of $\alpha$. Moreover, the isotropy group of a base point of $\mathcal{O}_{\delta}$ equals $\left(R_{u}\left(P_{I}\right) \times R_{u}\left(P_{I}^{-}\right)\right)\left(G_{\delta} \times G_{\delta}\right) \operatorname{diag} L_{\alpha}$, where $I=I(\delta) \cap\{\alpha\}$, and $G_{\delta} \subset L_{I(\delta)}$ is the connected kernel of the character $\mu-\lambda$ (or of $\alpha$ ). Thus, $\mathcal{O}_{\delta}$ fibers over $G / P_{I(\delta)} \times G / P_{I(\delta)}^{-}$with fiber $L_{\alpha} / \operatorname{ker}(\alpha)^{0}$, isomorphic to $\mathrm{SL}_{2}$ or $\mathrm{PGL}_{2}$.

6.3. Finally, as examples of reductive varieties of rank 2, we consider embeddings of the group $\mathrm{GL}(2)$. Let $B, B^{-}$be the opposite Borel subgroups of upper, respectively lower triangular matrices; then $T=B \cap B^{-}$is the maximal torus of diagonal matrices. Via the diagonal coefficients, the weight lattice $\Lambda$ identifies to $\mathbb{Z}^{2}$; then the unique simple root is $\alpha=(1,-1)$, the unique non-trivial element of the Weyl group is the reflection with respect to the diagonal of $\mathbb{R}^{2}$, and the positive Weyl chamber consists of all points below the diagonal.

Thus, projective embeddings of GL(2) correspond to lattice polygons in $\mathbb{R}^{2}$, symmetric with respect to the diagonal. Further, orbit closures in such embeddings correspond to the following four types of $W$-admissible faces $\varphi$ :

(i) $\varphi$ is an edge entirely below the diagonal. Then both sets $J(\varphi), K(\varphi)$ are empty, so that $G_{\varphi}=T_{\varphi}=T$. The isotropy group of a base point of the orbit $\mathcal{O}_{\varphi}$ is the product of $U \times U^{-}$with a torus of dimension 3 . The corresponding slice is an affine line, and the link is just a point.

(ii) $\varphi$ is an edge, symmetric with respect to the diagonal. Then $J(\varphi)=\emptyset, K(\varphi)=\{\alpha\}$, and $G_{\varphi}=T_{\varphi}$ is a torus of dimension 1. The isotropy group of $\mathcal{O}_{\varphi}$ is the product of diag GL(2) with $T_{\varphi} \times 1$. Again, the slice is a line, and the link is a point.

(iii) $\varphi$ is a vertex below the diagonal. Then $J(\varphi)=K(\varphi)=\emptyset, G_{\varphi}=T_{\varphi}=T$, and the isotropy group of $\mathcal{O}_{\varphi}$ is $B \times B^{-}$. So this orbit is isomorphic to $\mathbb{P}^{1} \times \mathbb{P}^{1}$. The slice is an affine toric variety of dimension 2 , so that the link is $\mathbb{P}^{1}$.

(iv) $\varphi$ is a vertex on the diagonal. Then $J(\varphi)=\{\alpha\}, K(\varphi)=\emptyset, T_{\varphi}=T$, and $G_{\varphi}=$ $\mathrm{GL}(2)$. The isotropy group of $\mathcal{O}_{\varphi}$ is the whole GL(2) $\times \mathrm{GL}(2)$, so that $\mathcal{O}_{\varphi}$ is a fixed point. The slice is the affine embedding of GL(2) associated with the tangent cone to $\delta$ at its vertex $\varphi$. The corresponding link is a projective embedding of the quotient of GL(2) by a non-trivial central torus, that is, of PGL(2). It follows that this link is the projective space $\mathbb{P}^{3}$, the projectivization of the space of $2 \times 2$ matrices.

\section{References}

[AB1] Alexeev, V., Brion, M.: Stable reductive varieties I: Affine varieties. Invent. Math. 154, 227-274 (2004) MR 2076923 
[AB2] Alexeev, V., Brion, M.: Stable reductive varieties II: Projective case. Adv. Math. 184, 380-408 (2003) Zbl pre02081972 MR 2054021

[BBFK1] Barthel, G., Brasselet, J.-P., Fieseler, K.-H., Kaup, L.: Equivariant intersection cohomology of toric varieties. In: Algebraic Geometry: Hirzebruch 70 (Warsaw, 1998), Contemp. Math. 241, Amer. Math. Soc., Providence, RI, 45-68 (1999) Zbl 0970.14028 MR 2001i:14030

[BBFK2] Barthel, G., Brasselet, J.-P., Fieseler, K.-H., Kaup, L.: Combinatorial intersection cohomology for fans. Tohoku Math. J. (2) 54, 1-41 (2002) Zbl pre01775132 MR 2003a: 14032

[BBFK3] Barthel, G., Brasselet, J.-P., Fieseler, K.-H., Kaup, L.: Combinatorial duality and intersection product: a direct approach. arXiv: math.AG/0309352

[BL] Bressler, P., Lunts, V.: Intersection cohomology on nonrational polytopes. Compositio Math. 135, 245-278 (2003) Zbl 1024.52005 MR 2004b:52016

[BJ] Brion, M., Joshua, R.: Vanishing of odd dimensional intersection cohomology II. Math. Ann. 321, 399-437 (2001) Zbl 0997.14005 MR 2003i:14022

[BP] Brion, M., Peyre, E.: The virtual Poincaré polynomials of homogeneous spaces. Compositio Math. 134, 319-335 (2002) Zbl pre01879569 MR 2004a:14051

[DeLo] Denef, J., Loeser, F.: Weights, exponential sums, intersection cohomology and Newton polyhedra. Invent. Math. 106, 275-294 (1991) Zbl 0763.14025 MR 93a:14019

[DiLe] Dimca, A., Lehrer, G.: Purity and equivariant weight polynomials. In: Algebraic Groups and Lie Groups, Austral. Math. Soc. Lecture Ser. 9, Cambridge Univ. Press, 161-181 (1997) Zbl 0905.57022 MR 99h:14023

[Fi] Fieseler, K. H.: Rational intersection cohomology of projective toric varieties. J. Reine Angew. Math. 413, 88-98 (1991) Zbl 0716.14006 MR 92m:14028

[Fu] Fulton, W.: Introduction to Toric Varieties. Ann. Math. Stud. 131, Princeton Univ. Press (1993) Zbl 0813.14039 MR 94g:14028

[Jo] Joshua, R.: Equivariant Riemann-Roch for $G$-quasi-projective varieties. $K$-theory 17, 1-35 (1999) Zbl 0926.19002 MR 2000e:19007

[Ka] Karu, K.: Hard Lefschetz theorem for non-rational polytopes. arXiv: math.AG/0112087

[Kn] Knop, F.: The asymptotic behavior of invariant collective motion. Invent. Math. 116, 309-328 (1994) Zbl 0802.58024 MR 94m:14063

[Lu] Luna, D.: Variétés sphériques de type A. Publ. Math. I.H.E.S. 94, 161-226 (2001) Zbl pre01874458 MR 2003f:14056

[St1] Stanley, R.: Generalized $h$-vectors, intersection cohomology of toric varieties, and related results. In: Adv. Stud. Pure Math. 11, North-Holland, 187-213 (1987) Zbl 0652.52007 MR 89f:52016

[St2] Stanley, R.: Subdivisions and local $h$-vectors. J. Amer. Math. Soc. 5, 805-851 (1992) Zbl 0768.05100 MR 93b:52012

[Su] Sumihiro, H.: Equivariant completion. J. Math. Kyoto Univ. 14, 1-28 (1974) Zbl 0277.14008 MR 49\#2732 